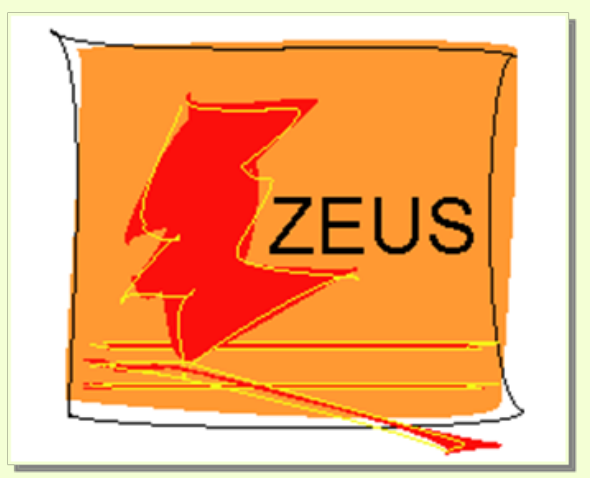

\title{
ZEUS Results
}

E. Tassi - Calabria Univ. and INFN

DIS 2009 Workshop - Madrid

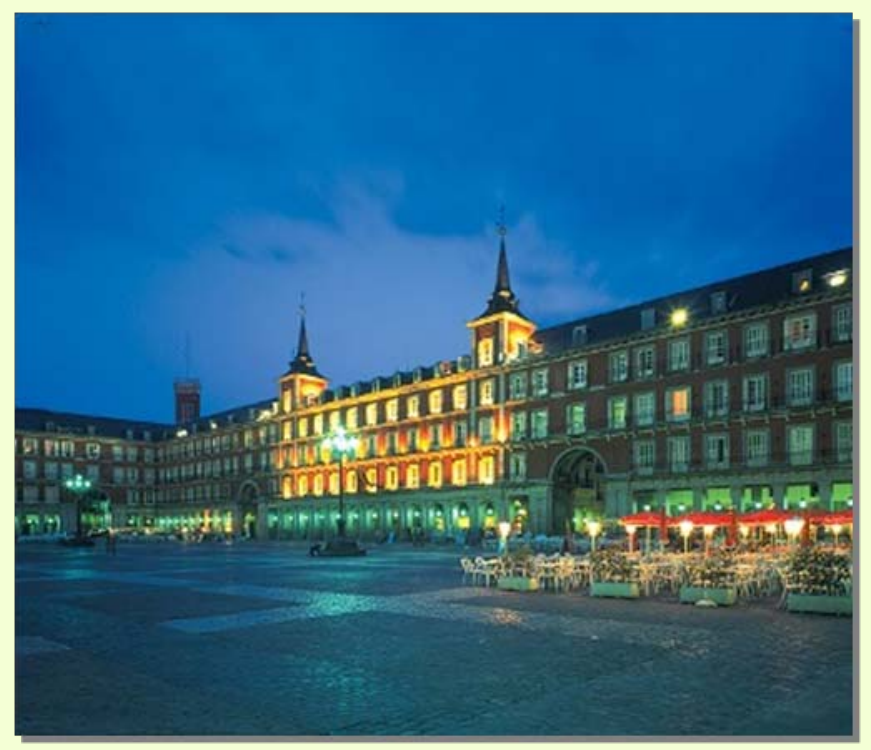




\section{Outline}

- Inclusive Measurements and Proton Structure

* HERA-I H1-ZEUS combined cross sections and HERAPDF0.2

- HERA-II Low $Q^{2} \mathrm{NC}^{+} \mathrm{p}$ Cross sections, $\mathrm{F}_{2}$ and $\mathrm{F}_{\mathrm{L}}$

- HERA-II High-Q ${ }^{2}$ NC/CC e-p and CC e+p cross sections

* ZEUS 2009 Proton's PDFs

* Inclusive diffraction and dPDFs

- Exclusive processes and pQCD

* Jet production in NC DIS and $\alpha_{s}$

* Subjets in NC DIS

* Semileptonic Charm and Beauty in DIS

* Beauty in yp

* Prompt-photon production 


\section{ZEUS Results for DIS09}

\section{Electroweak:}

- Single Top production

- Isolated Leptons

- Multi-leptons

Inclusive:

- FL

- High-Q2 NC/CC

- H1/ZEUS Combination

- HERAPDF0.2

\section{Hadronic Final States:}

- Jet Substructure

- KOsKOs

- Scaled momentum distributions

- Angular Correlations in 3-jets

- Jet Cross Sections in DIS and gp

- Prompt-photon production
Diffraction:
Stefano Antonelli

Gerhard Brandt

Monica Turcato

- Inclusive Diffraction

- Factorization Tests

- Leading Barions

- Vector Meson Production
Marta Ruspa

Wojtek Slominski

Graziano Bruni

Aharon Levy
Julia Grebeniuk

Amanda Cooper-Sarkar

Heavy Flavours:
Enrico Tassi - Charm production in DIS
Voica Radescu
- Beauty Production in DIS
- J/psi helicity distributions
- Charm Fragmentation

Elias Ron

David Saxon

John Morris

Steve Magill

Claire Gwenlan

Matthew Forrest 


\section{H1-ZEUS combined HERA I cross sections}

New combination based on the full HERA-I incl. data $L=240 \mathrm{pb}^{-1}$

Added since DIS08:

* Zeus 95-97 "low Q2"

*H1 95-00 "low Q2"

:H1 96-00 "Bulk"

Reduced systematic uncert. and $\mathrm{O}(1 \%)$ precision for:

$$
10<\mathrm{Q}^{2}<100 \mathrm{GeV}^{2}
$$

Used as single input to a new QCD analysis:

$$
\Rightarrow \text { HERAPDF0.2 }
$$

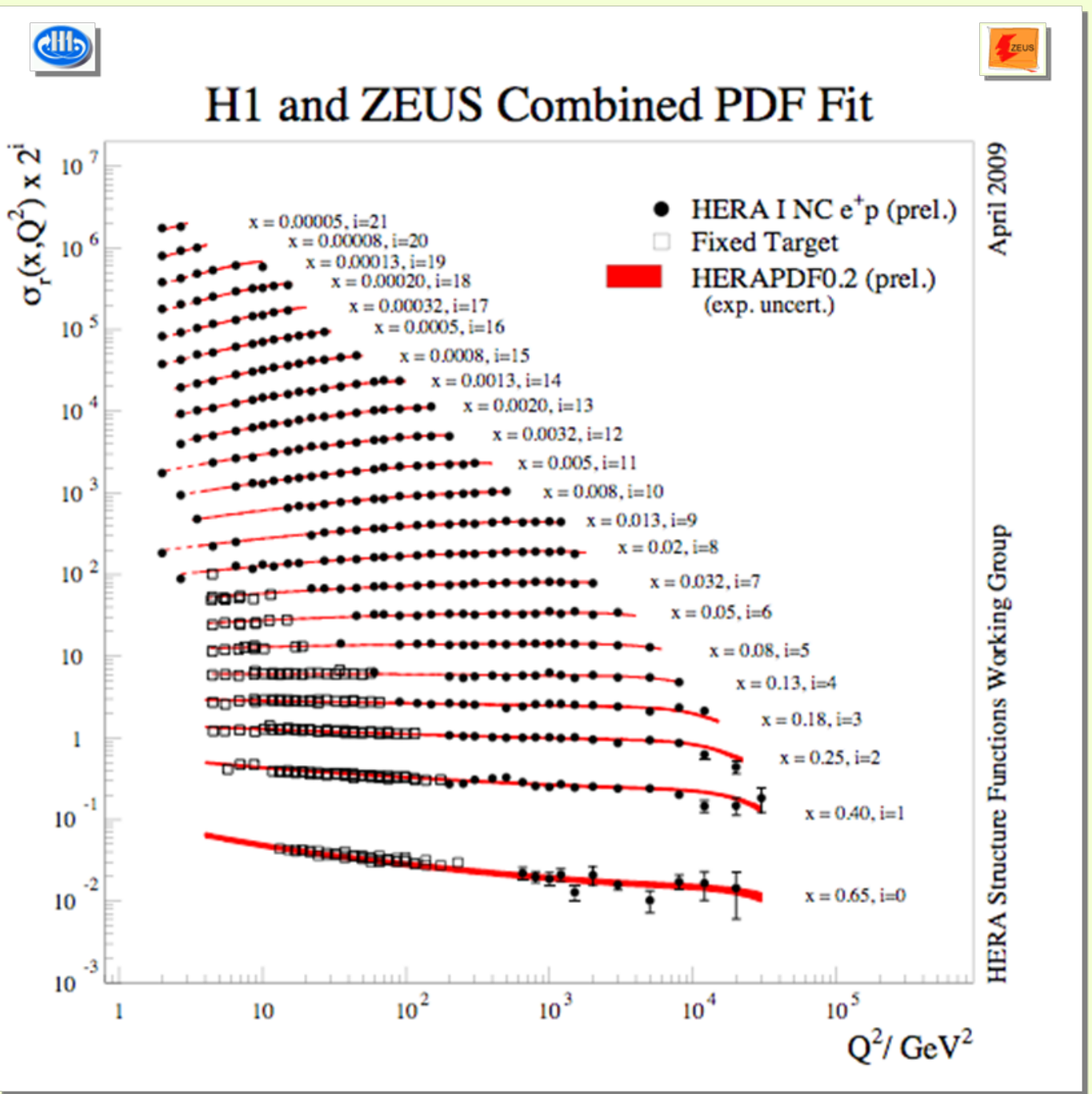

see E.T.[63] 


\section{New PDF Fit to the combined HERA-I data}

\section{HERAPDF0.2:}

* Very detailed study of PDFs uncert.

* Heavy Flavours (Roberts-Thorne)
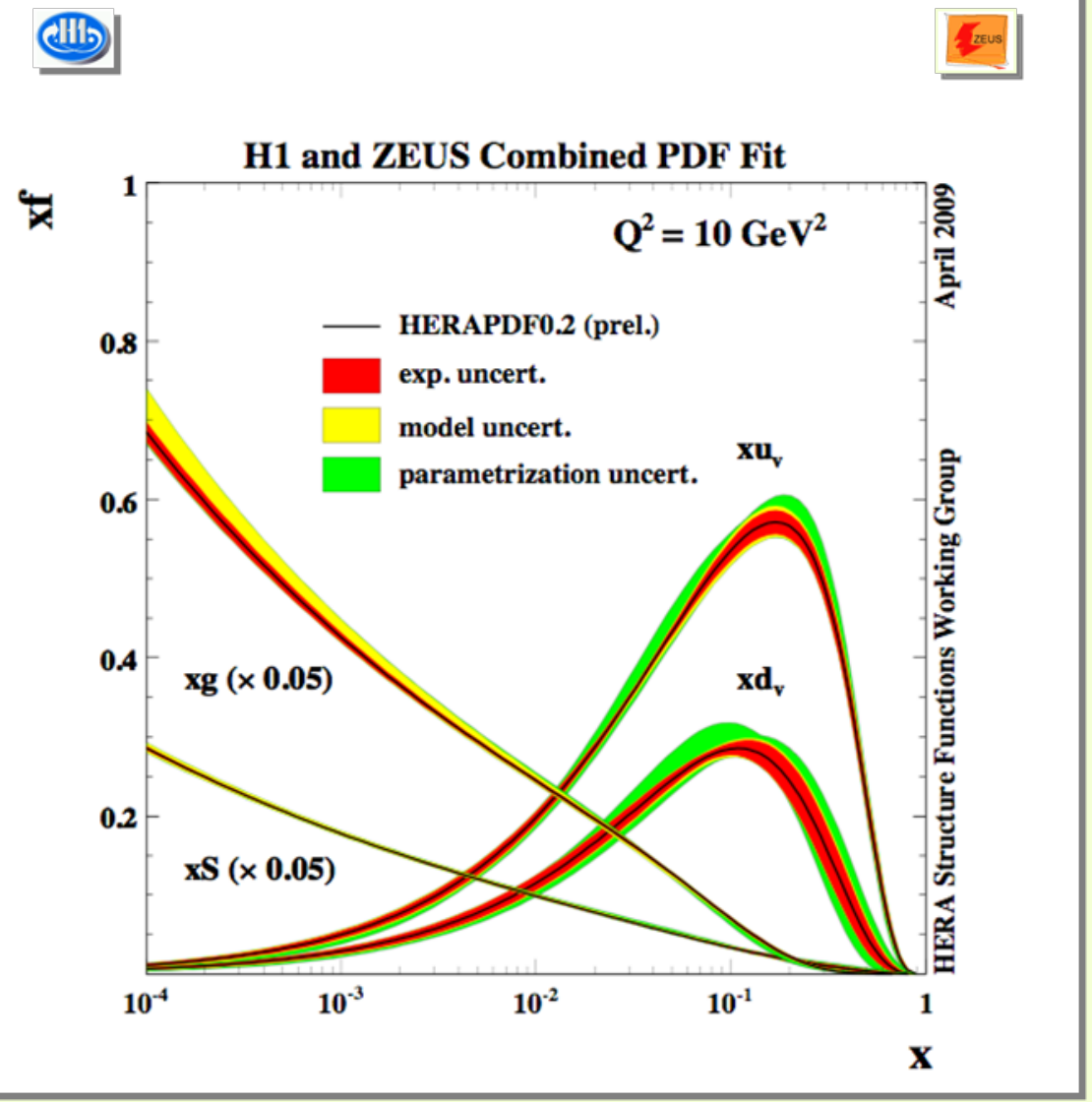

\section{H1 and ZEUS Combined PDF Fit}
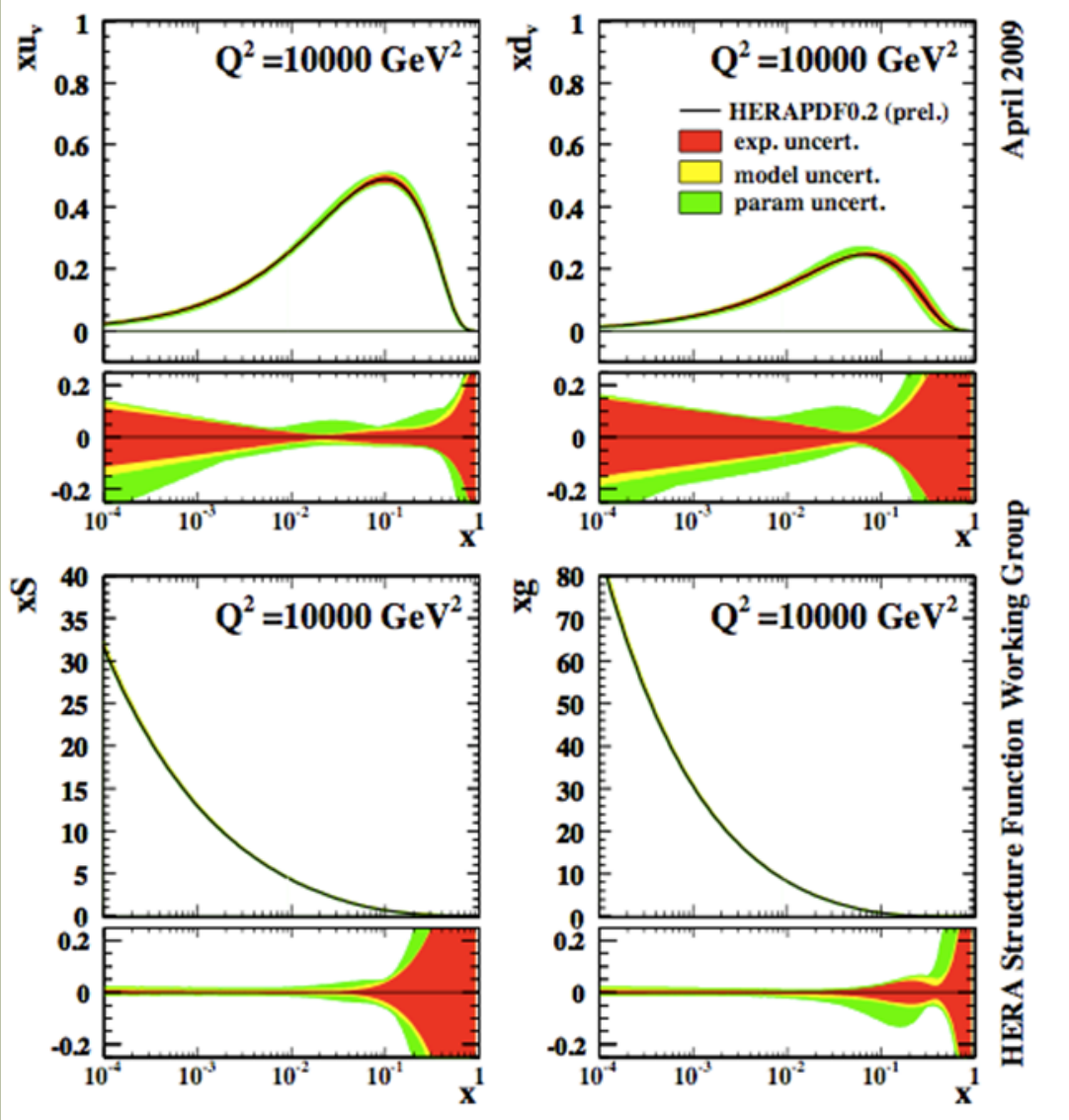

$x S, x g$ high precision at low- $x$

see V. Radescu[86] 


\section{6/07 NC e+p at low- mid-Q2 : "F $\mathrm{F}_{\mathrm{L}}$ runs"}

Three data sets collected with dedicated ZEUS DESY-09-046

triggers at the center of mass energies:

- $318 \mathrm{GeV}$ (HER) 44.5pb-1

- $251 \mathrm{GeV}$ (MER) $7.1 \mathrm{pb}^{-1}$

- $225 \mathrm{GeV}$ (LER) $13.9 \mathrm{pb}^{-1}$

Reduced cross sections measured in the kinematic region:

$$
\begin{aligned}
& 20<\mathrm{Q}^{2}<130 \mathrm{GeV}^{2} \\
& 5 \times 10^{-4}<x<0.007
\end{aligned}
$$

A precise measurement and an important input to QCD analyses

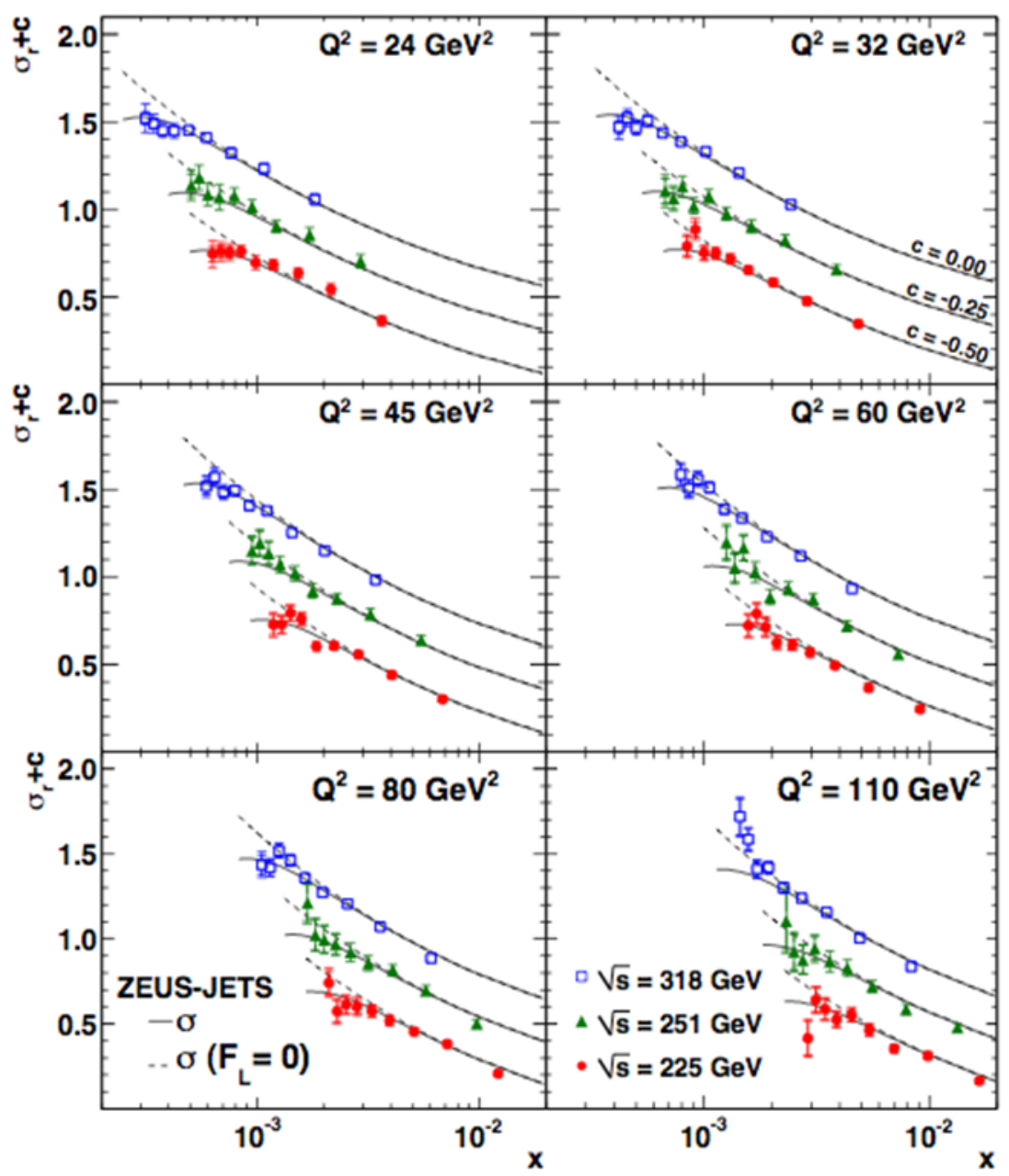


$\sigma_{r}\left(x, Q^{2}, y\right)=F_{2}\left(x, Q^{2}\right)-\frac{y^{2}}{Y_{+}} \cdot F_{L}\left(x, Q^{2}\right)$

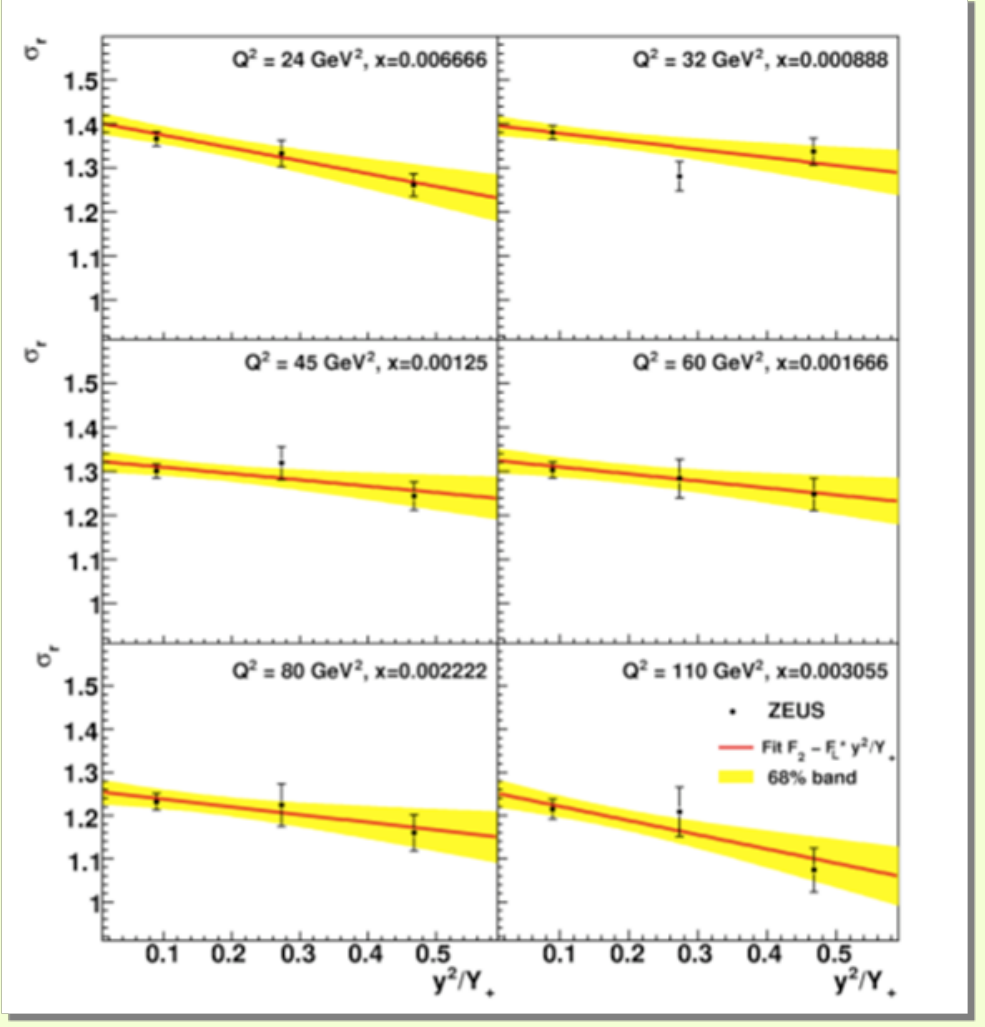

* Most precise $F_{2}$ from ZEUS in $\square \square \square \quad$ this kinematic

\section{region}

* Data support non-zero $\mathrm{F}_{\mathrm{L}}$

\section{$F_{2}$ and $F_{L}$}

\section{ZEUS}

\section{DESY-09-046}

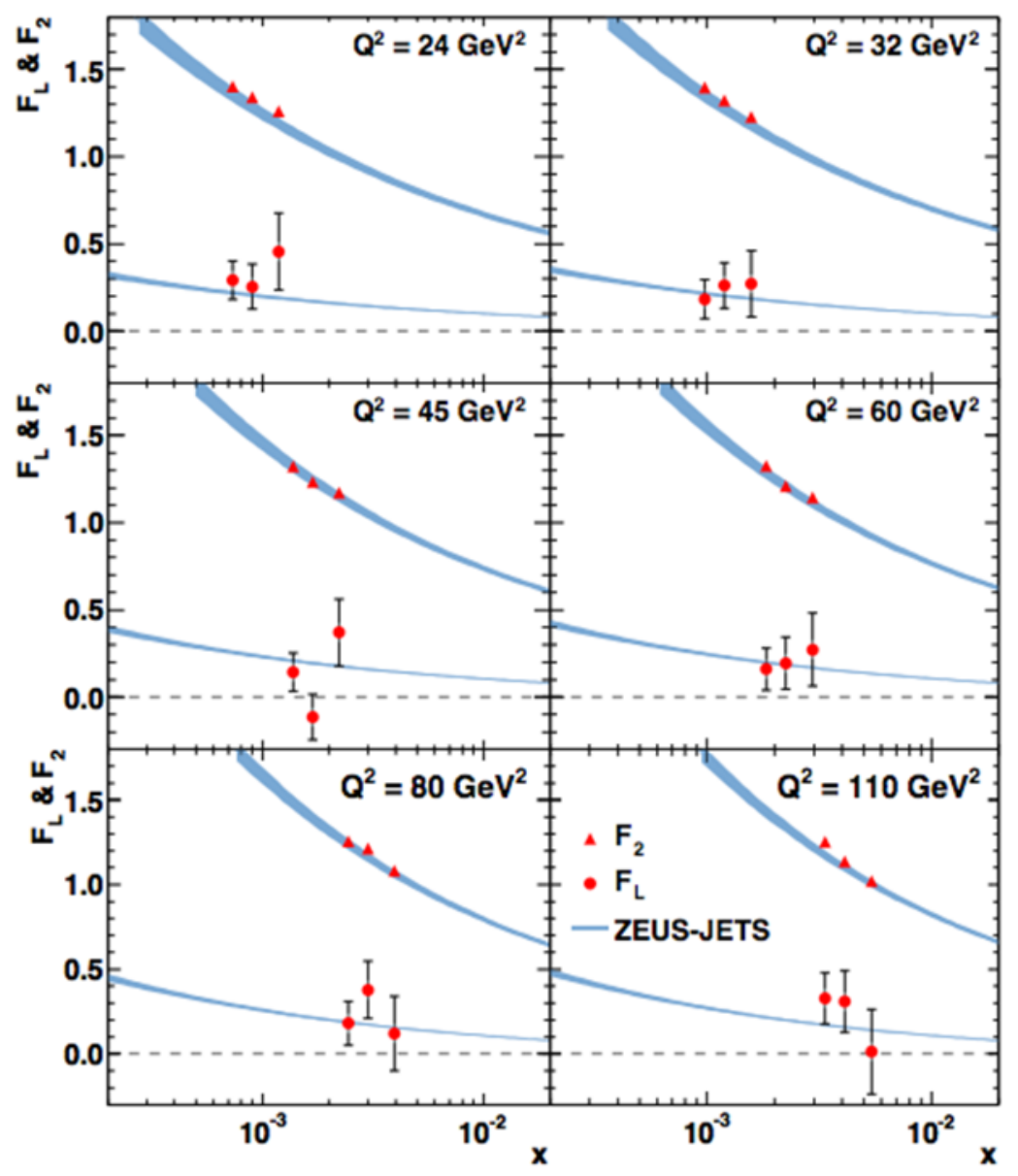




\section{NC/CC High-Q2 ${ }^{2}$ Cross sections at HERA}

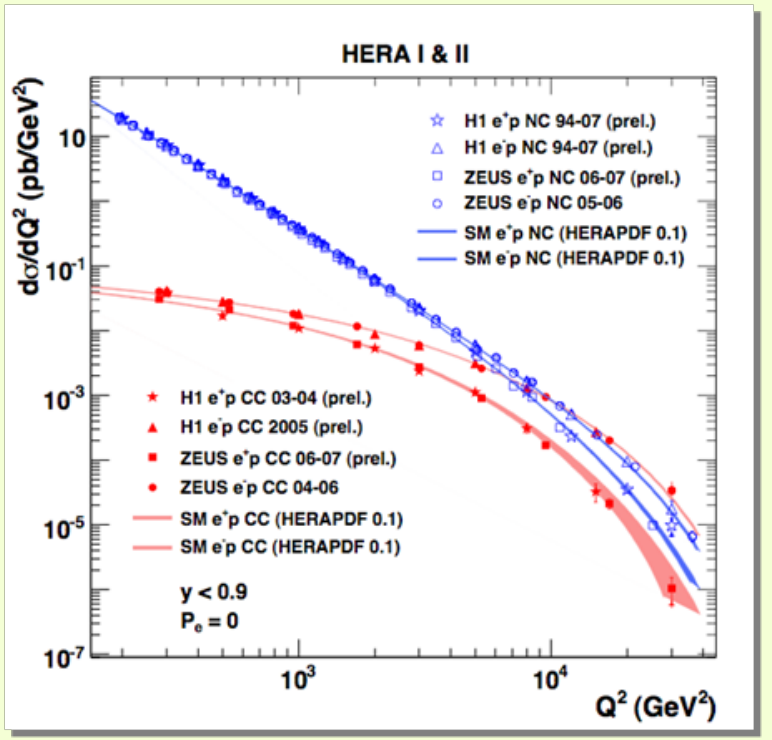

Important Electroweak Physics program:

* Electroweak unification

* Chiral nature of the SM

* Determination of light-quarks couplings

* Parity violation in DIS at high-Q $Q^{2}\left(10^{-18} \mathrm{~m}\right)$

Here will concentrate on their impact on the proton structure
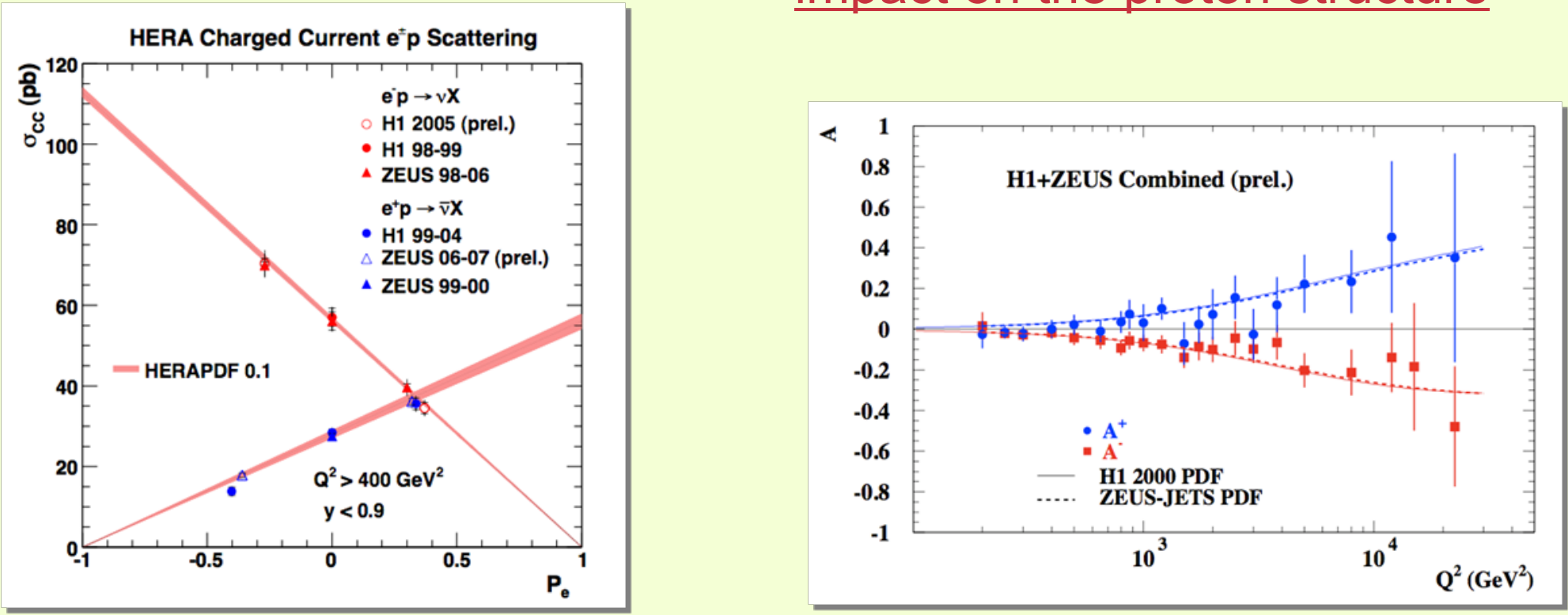


\section{High $Q^{2}$ NC e-p cross sections}

$\tilde{\sigma}^{e^{ \pm} p}=\frac{x Q^{4}}{2 \pi \alpha^{2}} \frac{1}{Y_{+}} \frac{d^{2} \sigma\left(e^{ \pm} p\right)}{d x d Q^{2}}=\tilde{F}_{2}\left(x, Q^{2}\right) \mp \frac{Y_{-}}{Y_{+}} x \tilde{F}_{3}\left(x, Q^{2}\right)$
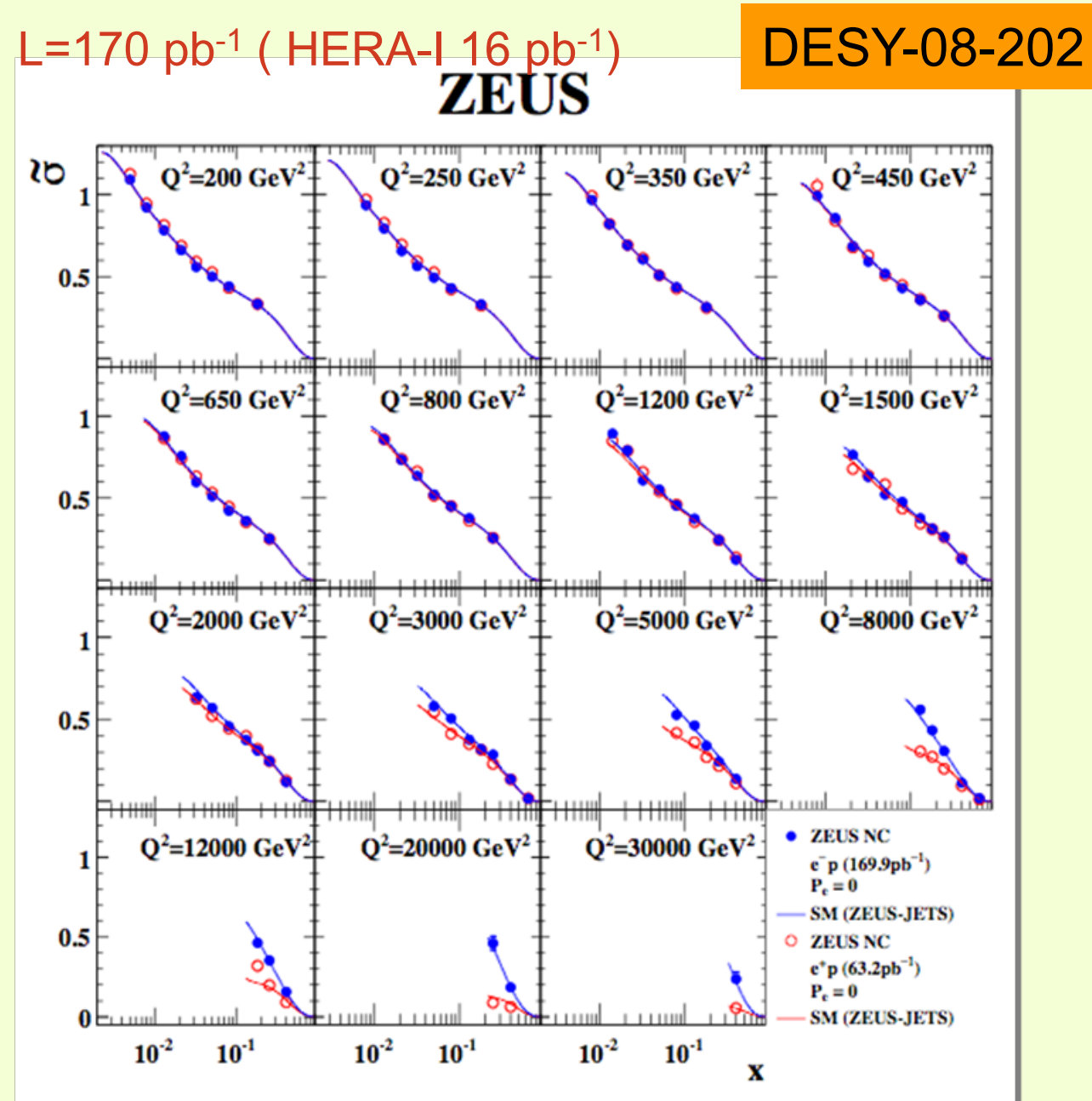

$x \tilde{F}_{3}=\frac{Y_{+}}{2 Y_{-}}\left(\tilde{\sigma}^{e^{-} p}-\tilde{\sigma}^{e^{+} p}\right)$

\section{ZEUS}

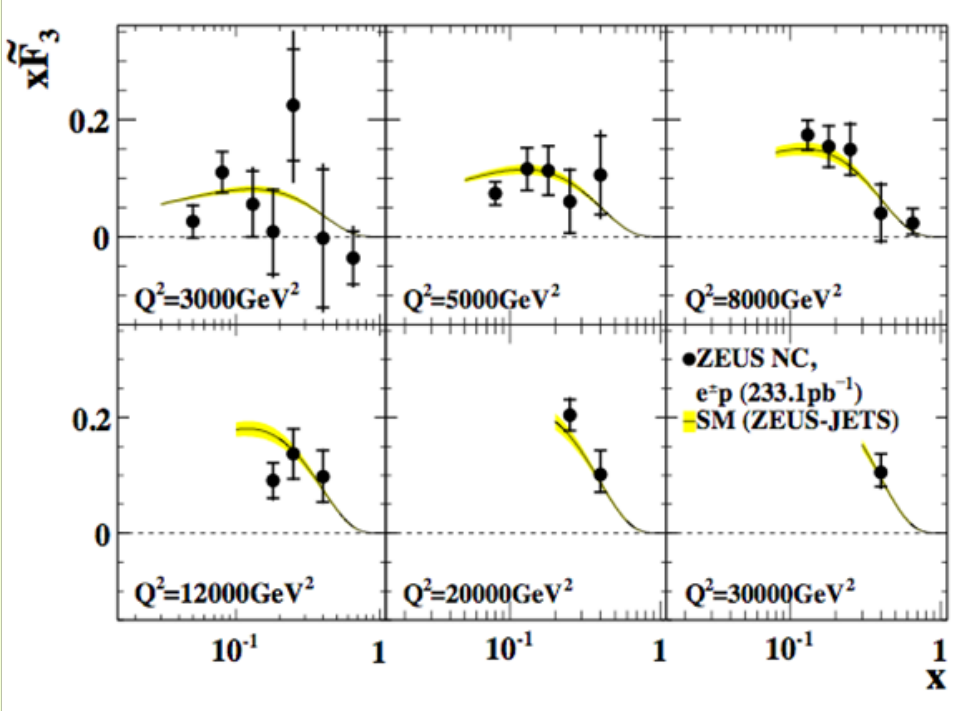

$x F_{3}^{\gamma Z}=2 x\left[e_{u} a_{u} u_{v}+e_{d} a_{d} d_{v}\right]=\frac{x}{3}\left(2 u_{v}+d_{v}\right)$

$\Rightarrow$ Improve $\mathrm{xu}_{\mathrm{v}}$ at high- $\mathrm{x}$

see A. Cooper-Sarkar[59] 


\section{High $\mathrm{Q}^{2}$ CC e-p cross sections}

$\tilde{\sigma}\left(e^{-} p \rightarrow \nu_{e} X\right)=x\left[u+c+(1-y)^{2}(\bar{d}+\bar{s})\right]$

DESY-08-177
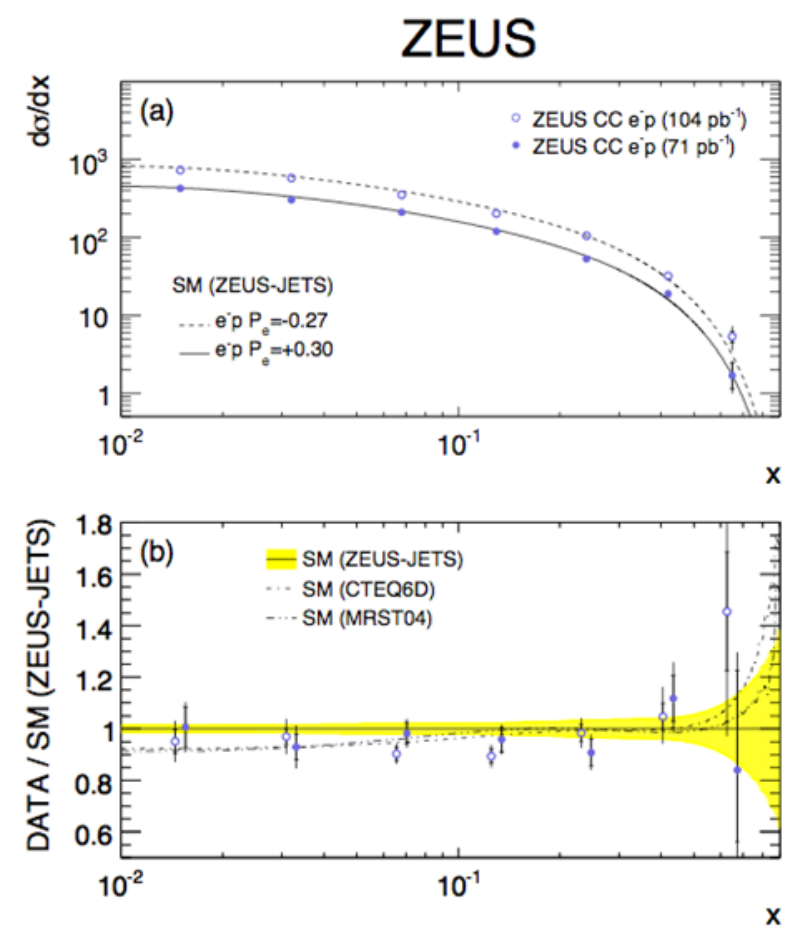

$\Rightarrow$ Improve $\mathrm{xu}_{\mathrm{v}}$ at high- $\mathrm{x}$

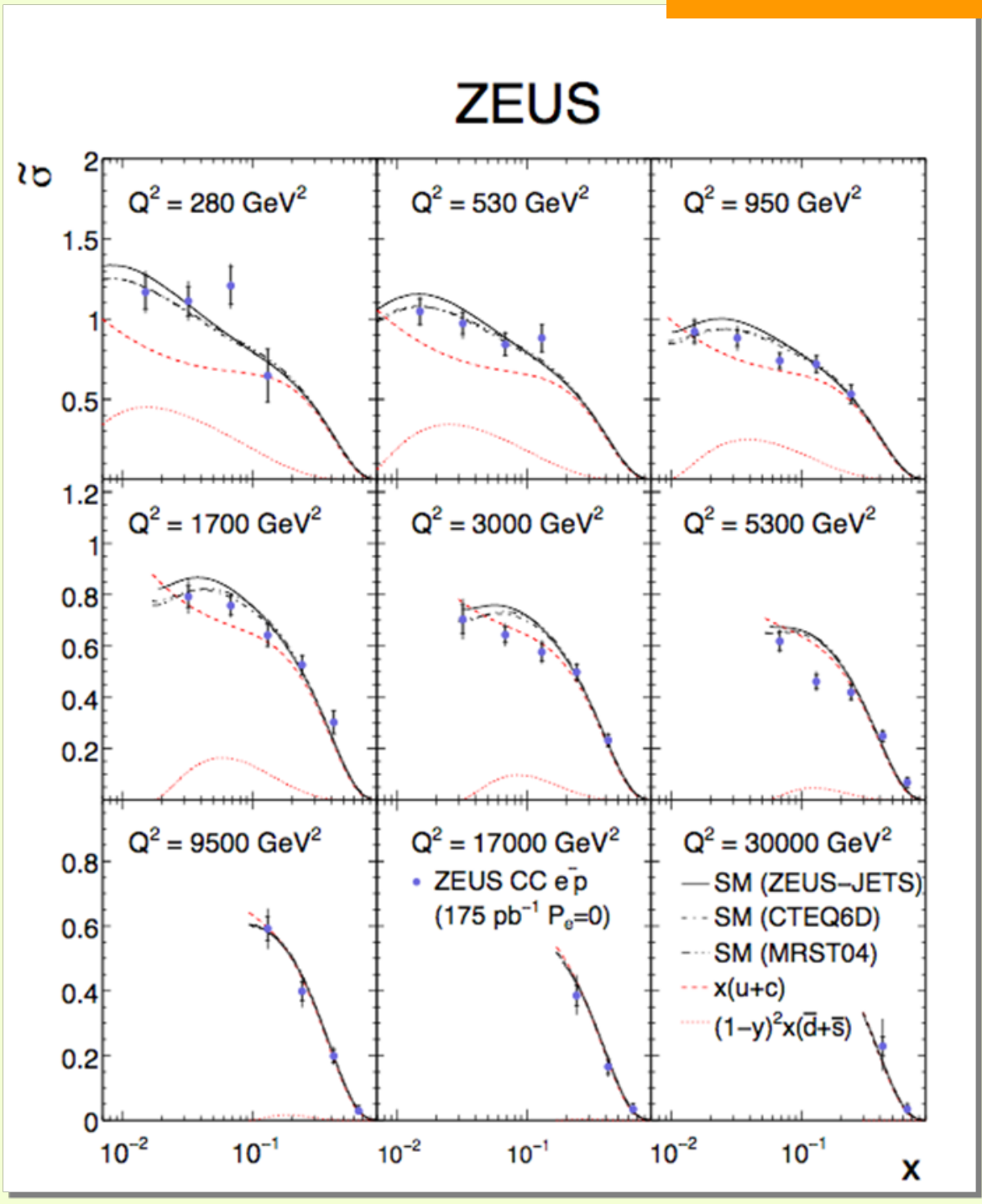




\section{High $\mathrm{Q}^{2} \mathrm{CC} \mathrm{e}^{+} \mathrm{p}$ cross sections}

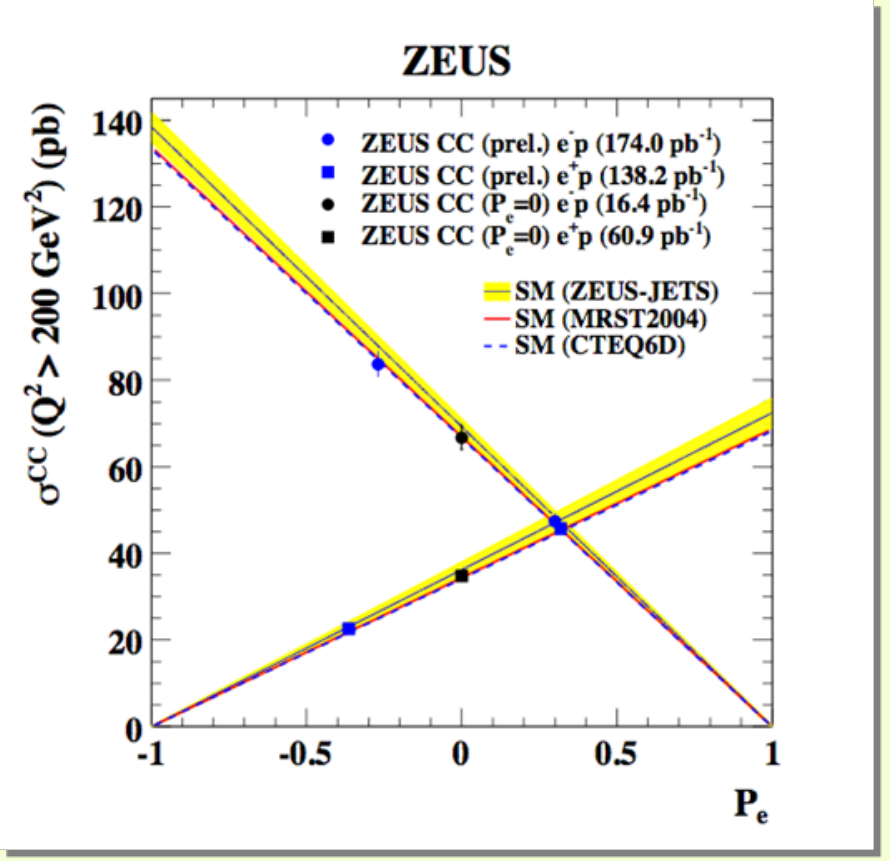

$\tilde{\sigma}\left(e^{-} p \rightarrow \nu_{e} X\right)=x\left[\left(\bar{u}+\bar{c}+(1-y)^{2}(d+s)\right]\right]$

$L=136 p^{-1}$

ZEUS

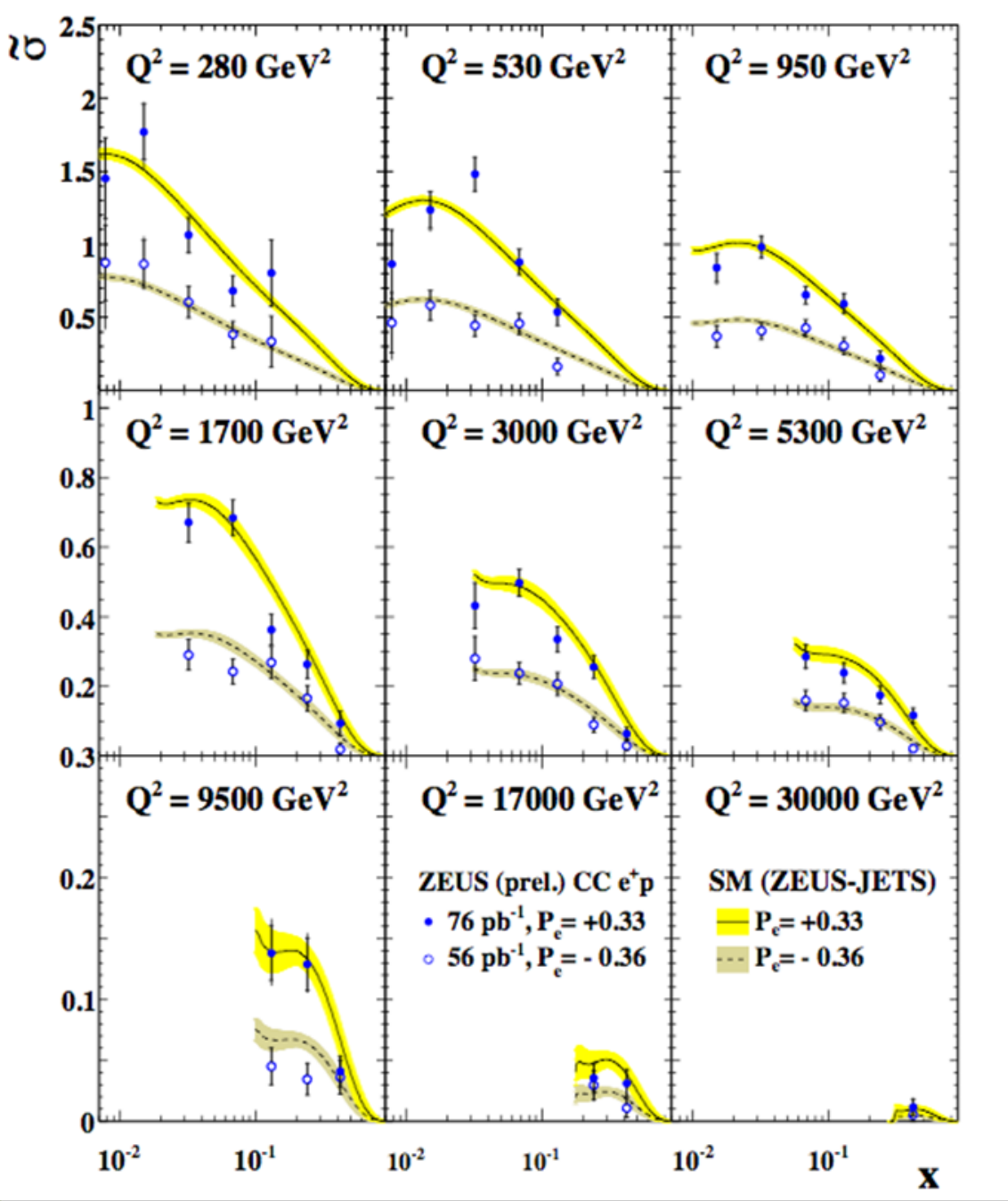




\section{Zeus 2009 Fit}

Use same approach as for the published ZEUS-JETS Fit to assess the impact of the new data:

$C C e^{ \pm} p, N C$ e-p and NC $e^{*} p$ H/M/LER
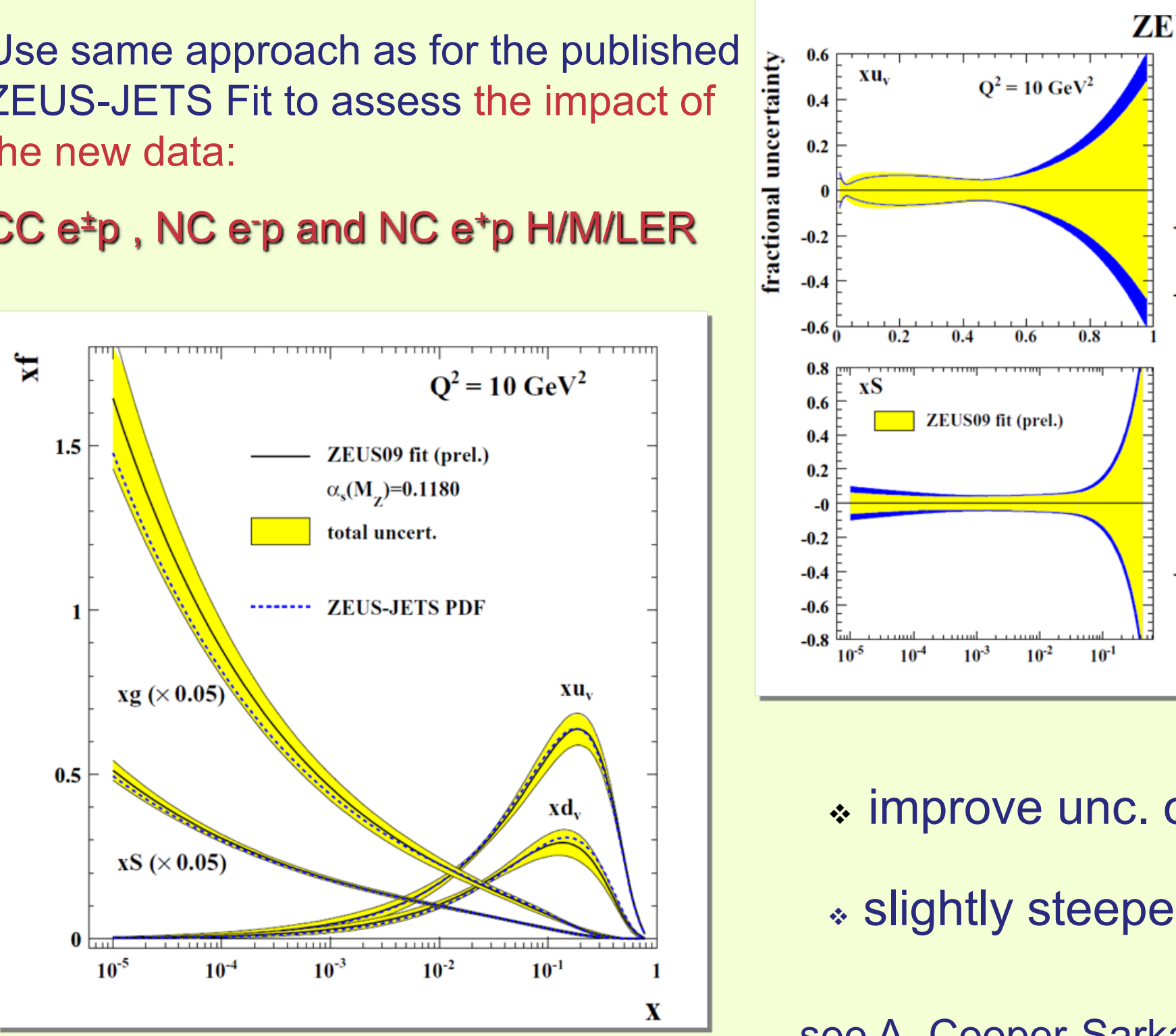

ZEUS
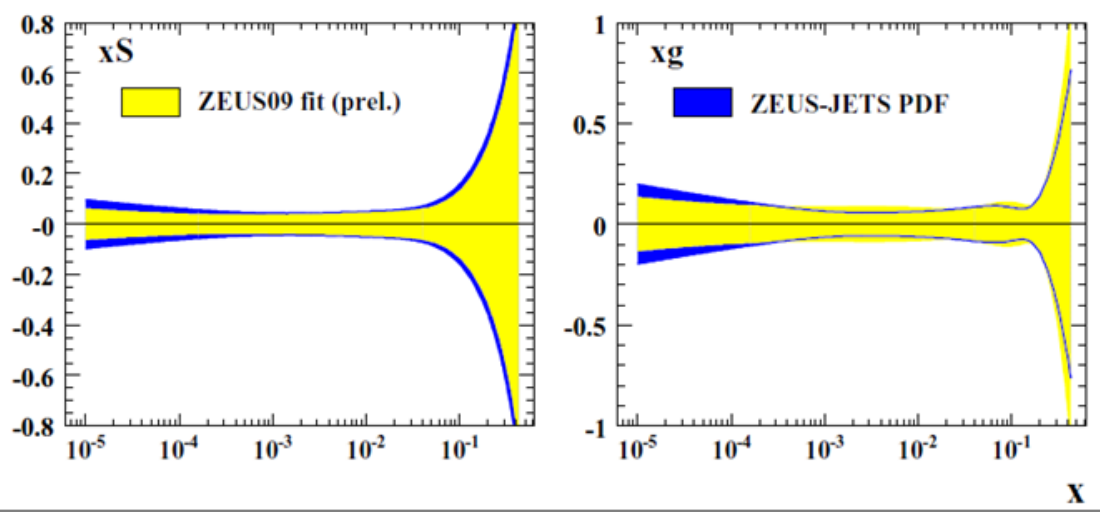

* improve unc. on $\mathrm{xu}_{\mathrm{v}}, \mathrm{xd}_{\mathrm{v}}$

* slightly steeper gluon

see A. Cooper-Sarkar[59] 


\section{Diffractive fit and dPDFs}

\section{LRG}

\section{ZEUS}

- ZEUS LRG 99.00 - ZEUS (prel.) DPDF S incl - - - ZEUS (prel.) DPDF S incl (extrapolated)

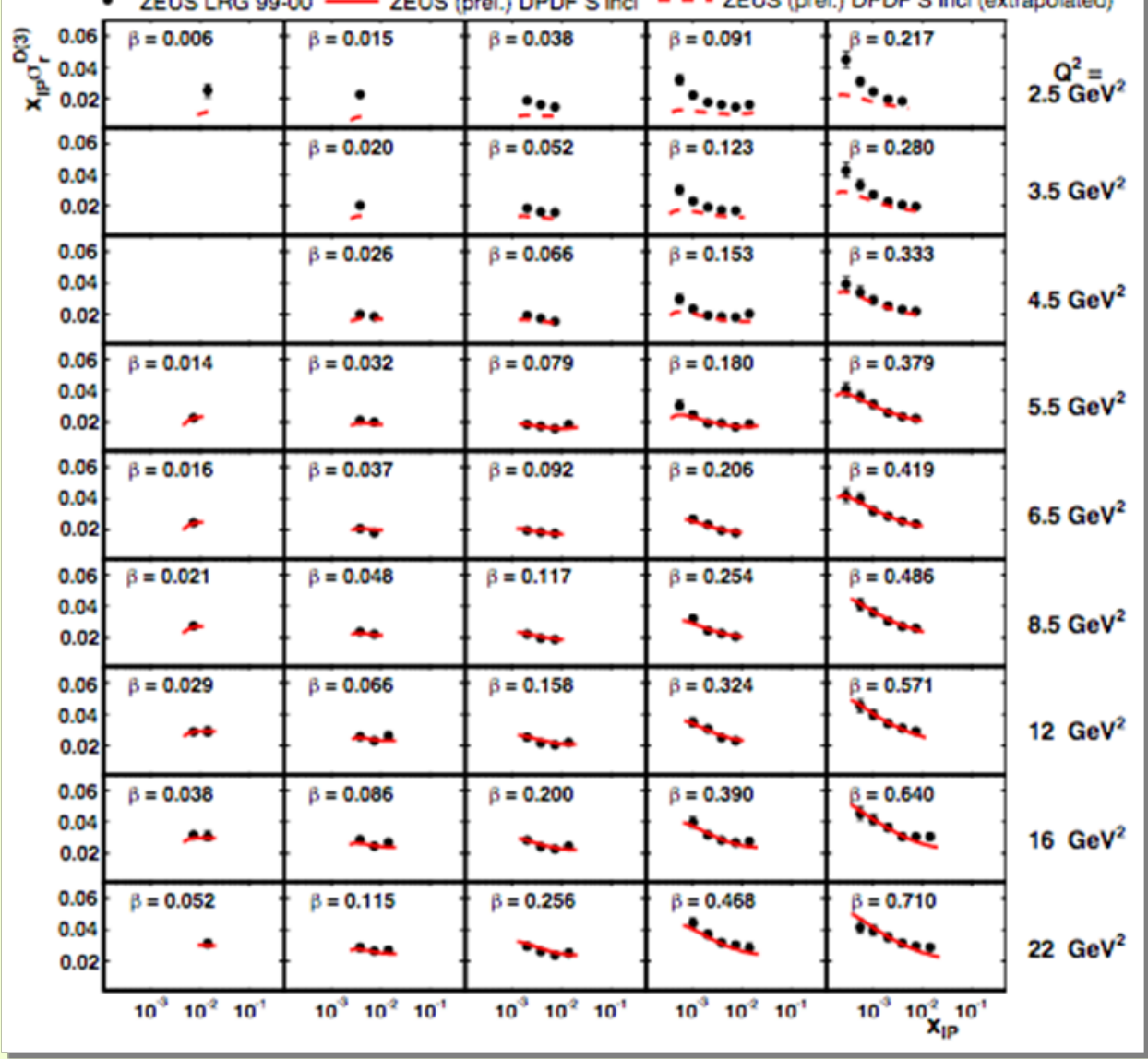

see W. Slominski[121]
* NLO QCD fit to incl. DIS data

* Comparison and fit to dijet in DIS

* Comparison to dijet in PHP

* Heavy Flavours (Roberts-Thorne)

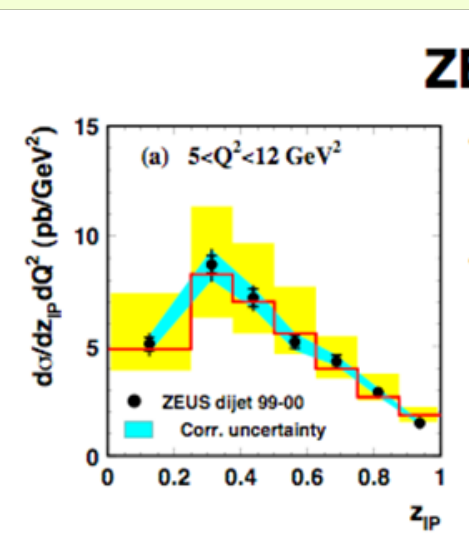

zeus Dijet
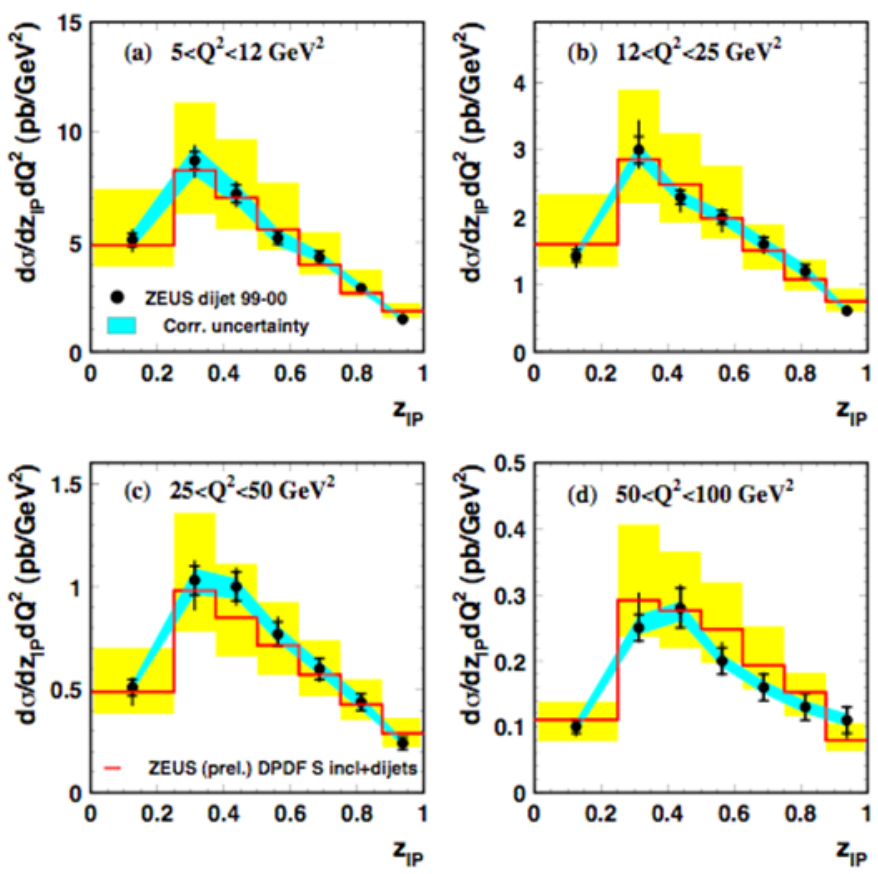


\section{Diffractive fit and dPDFs}

$\mathrm{zq}$

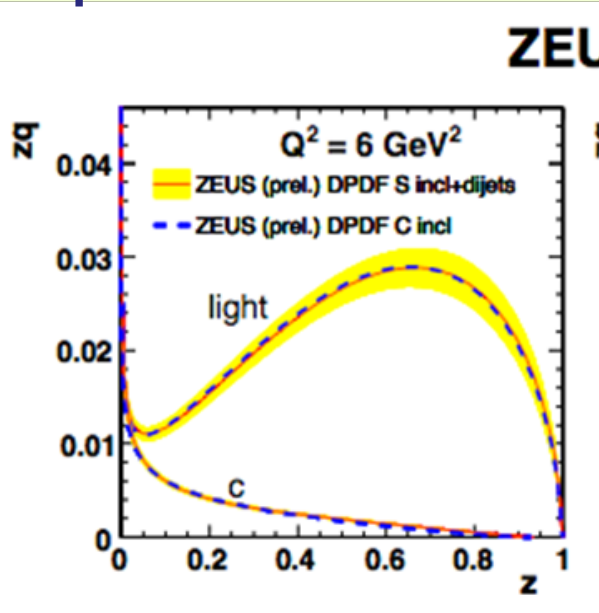

\section{ZEUS}
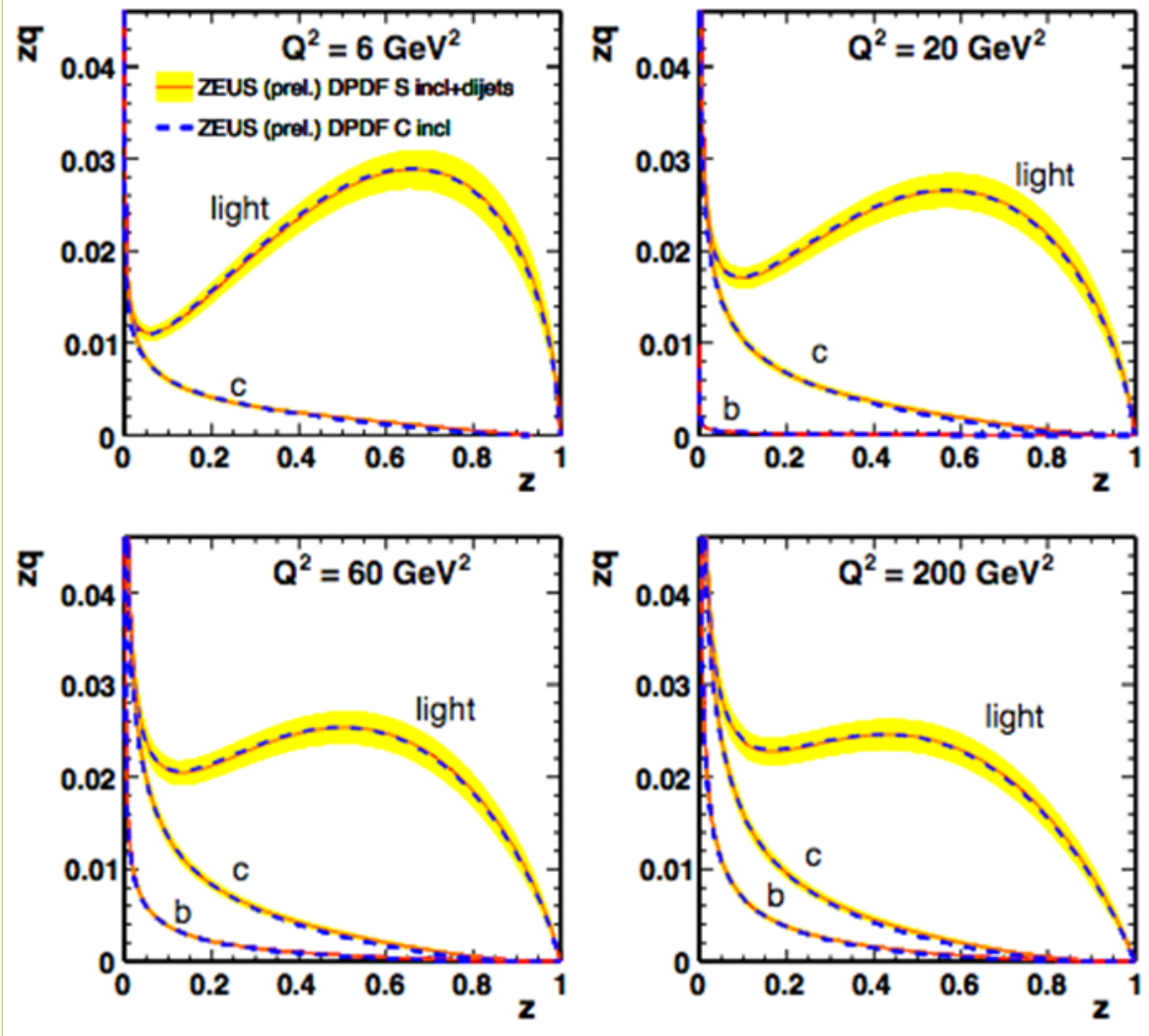

$\mathrm{xg}$

ZEUS

R
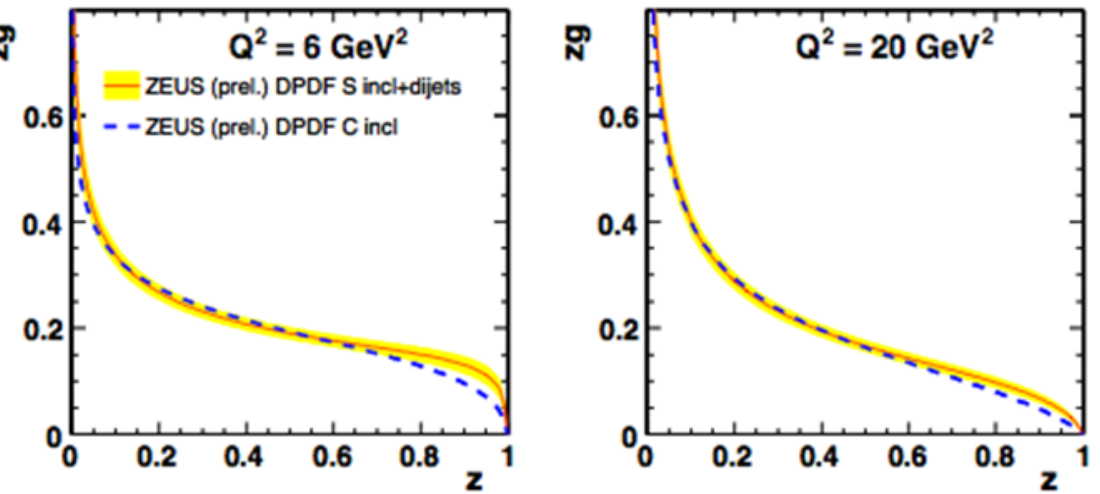

N

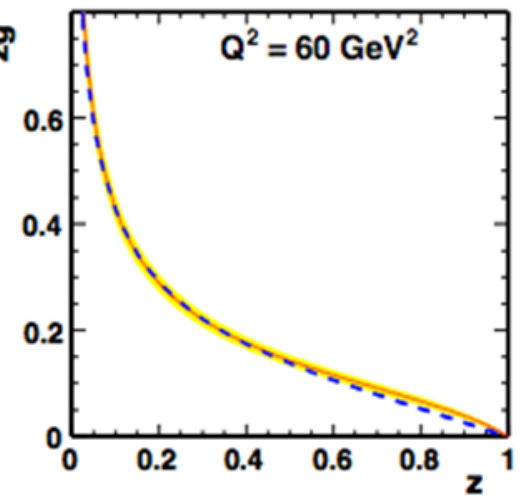

요

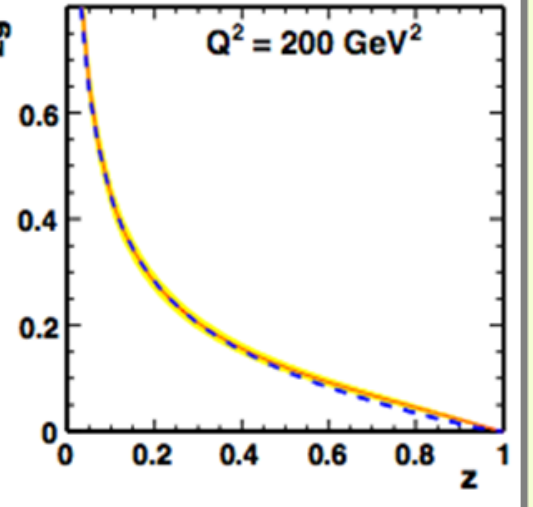




\section{Jet production in NC DIS}

\section{HERA-II e-p}

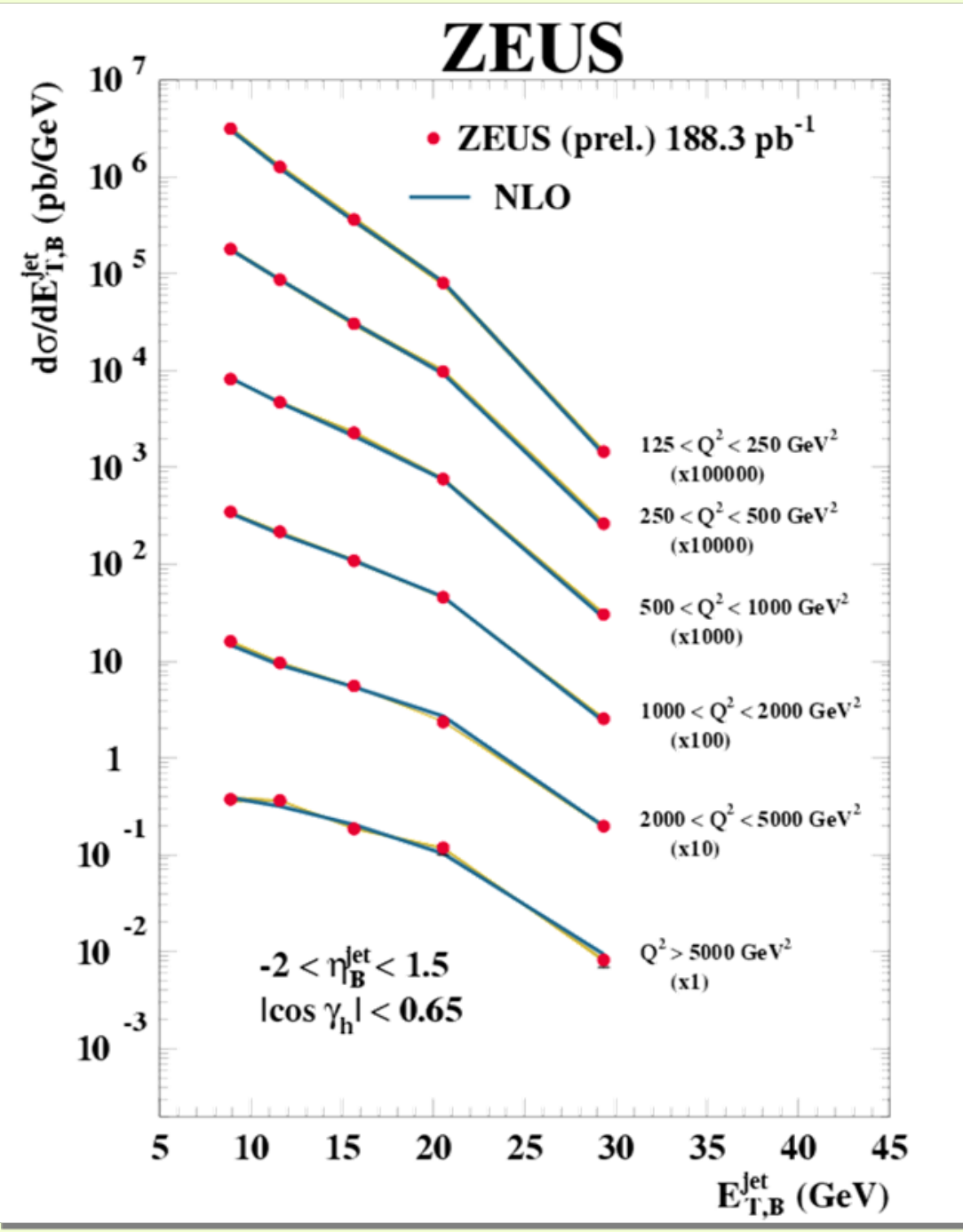

ZEUS

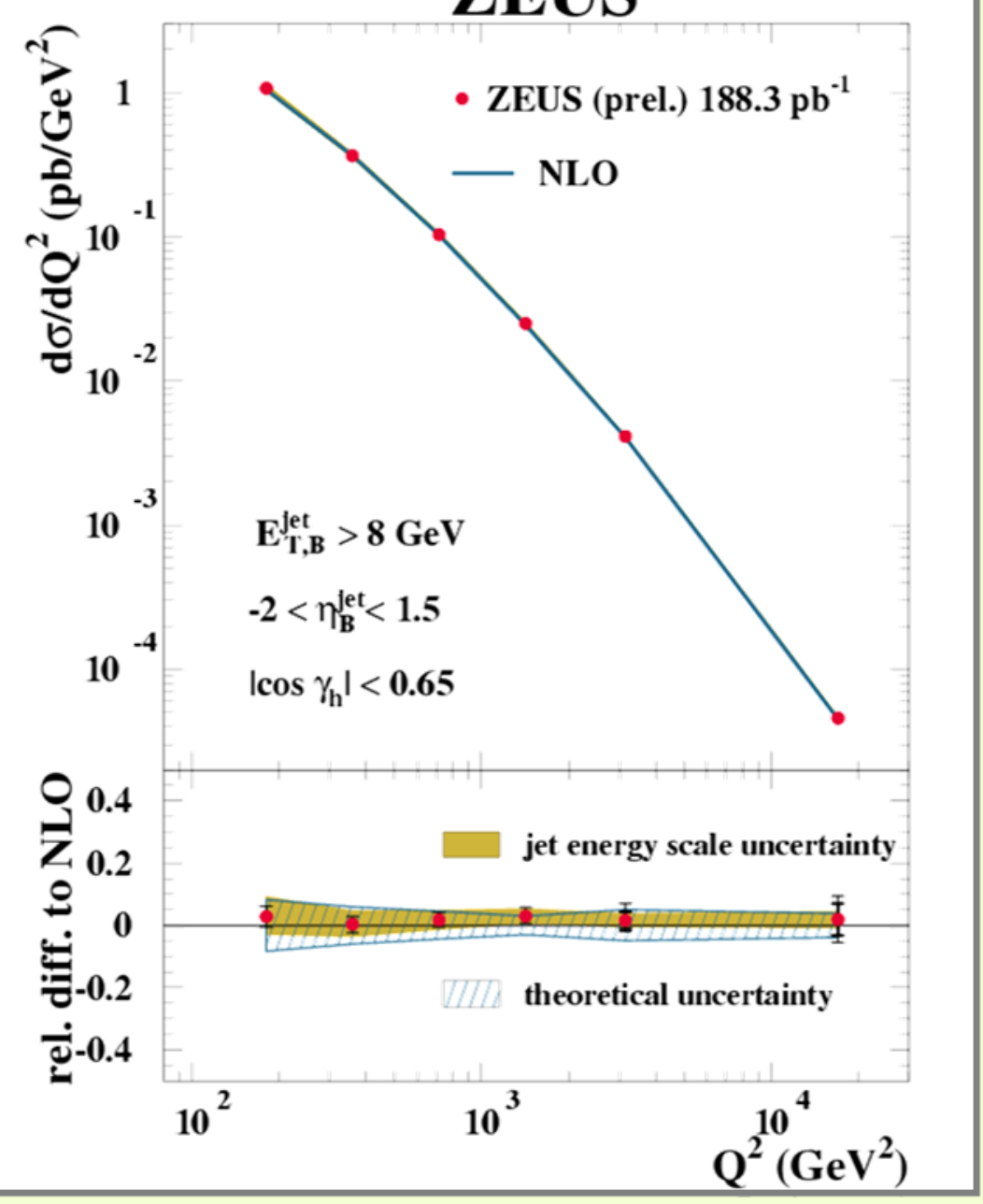

$\Rightarrow \alpha_{\mathrm{s}}$ determination 


\section{Strong coupling}

HERA-II NC DIS inclusive jets

(first determination from ZEUS with HERA-II data):

$\alpha_{s}\left(M_{z}\right)=0.1192 \pm 0.0009$ (stat. $)_{-0.0032}^{+0.0035}(\exp .)_{-0.0021}^{+0.0020}$ (th. $)(3.5 \%$ total $)$

Re-analysis of HERA-I yp incl. jets:

$\alpha_{s}\left(M_{z}\right)=0.1223 \pm 0.0001$ (stat. $)_{-0.0021}^{+0.0023}(\exp .)_{-0.0030}^{+0.0029}$ (th.) $(3.1 \%$ total)

: Improved exp. unc. from full HERA data

$\because$ Need NNLO

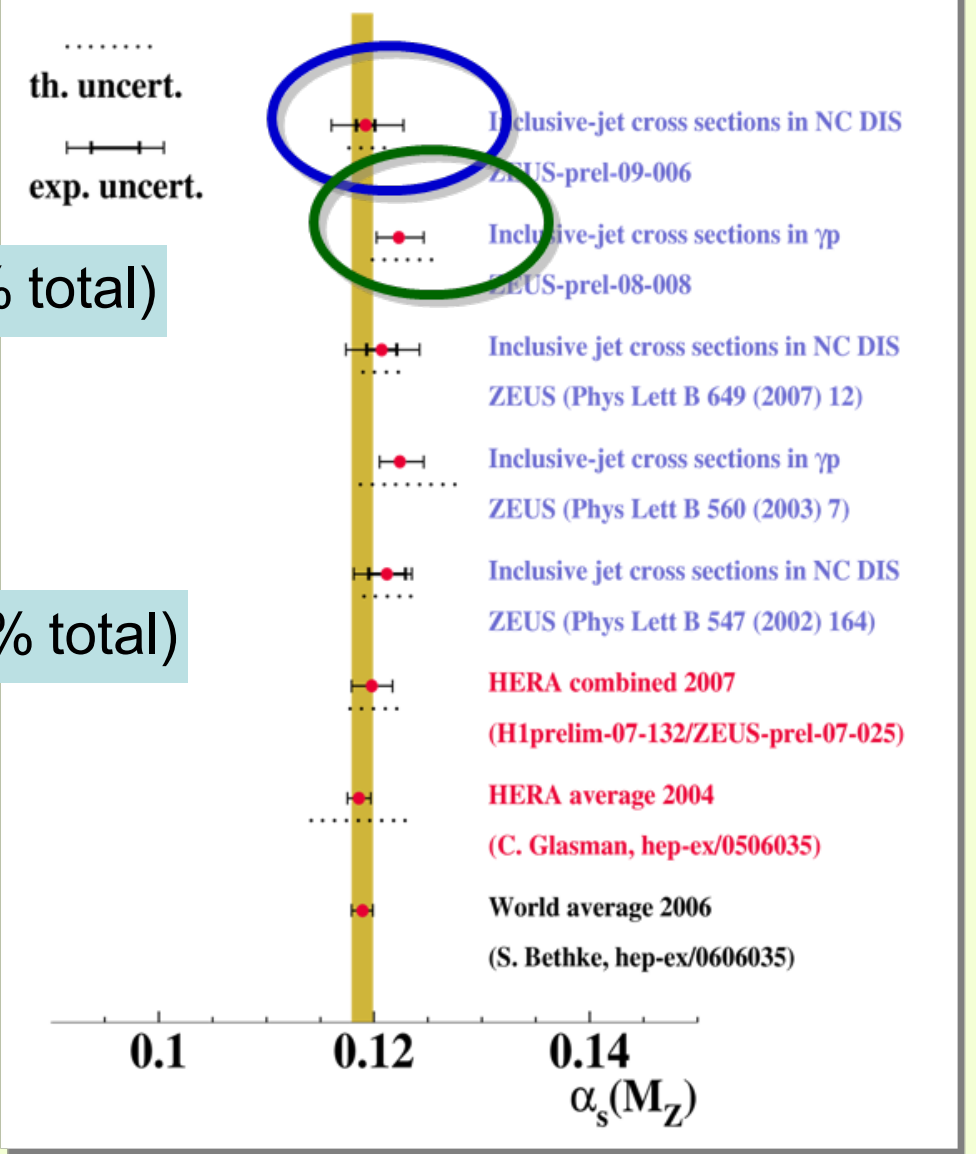




\section{Subjets}

DESY-08-178

Study transition parton $\Rightarrow$ hadrons

Two resolved subjets $\left(\mathrm{y}_{\text {cut }}=0.05\right)$ :

$\therefore$ Highest $\mathrm{E}_{\mathrm{T}}$ jet closer to the jet axis

* Lowest $\mathrm{E}_{\mathrm{T}}$ jet towards p-beam direction

New for DIS2009:

Three resolved subjets $\left(\mathrm{y}_{\text {cut }}=0.03\right)$

$\left(\mathrm{L}=334 \mathrm{pb}^{-1}\right)$

\section{ZEUS}

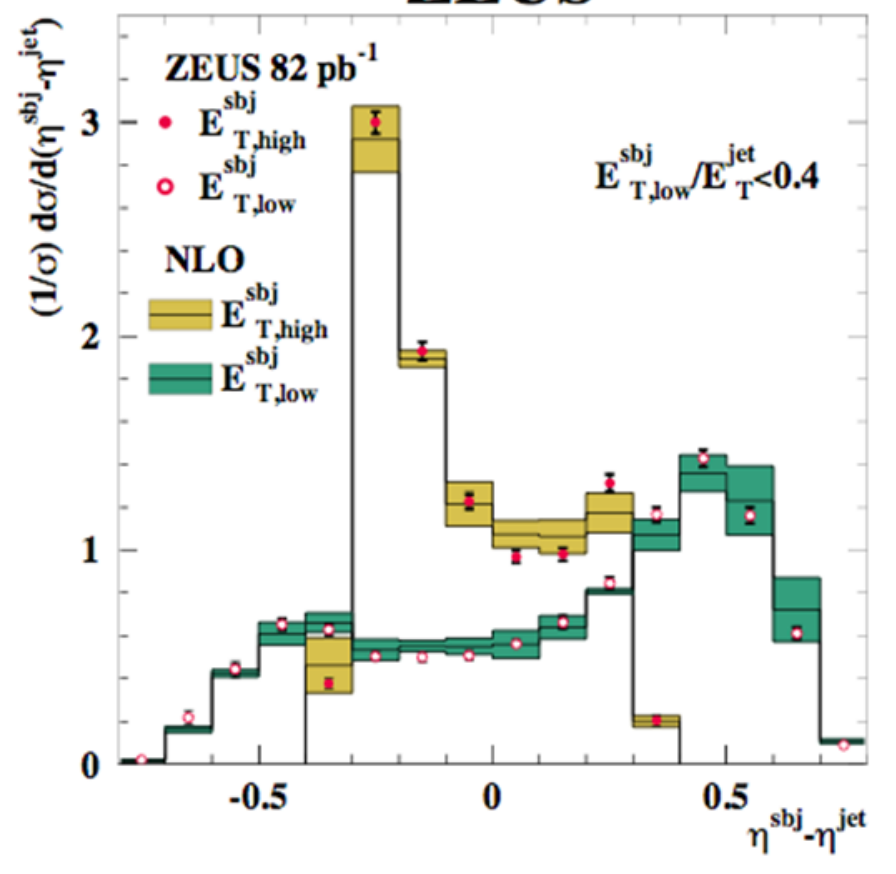

see E. Ron[240] 


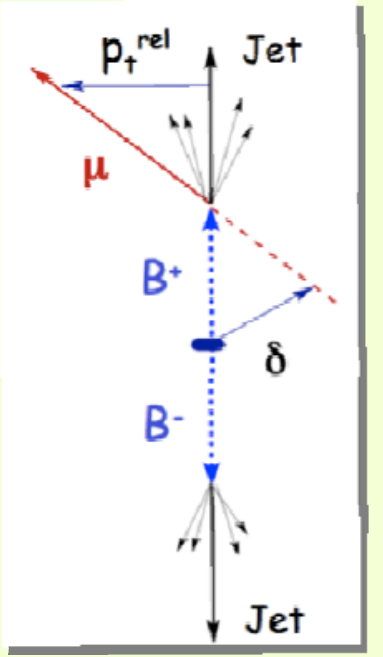

\section{Semileptonic Charm and Beauty}

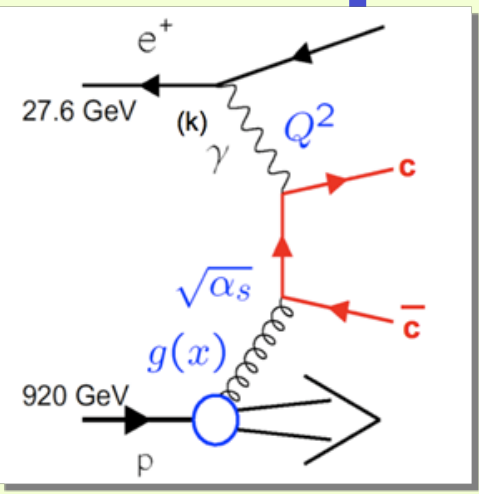

HERAIl e-p (2004/05) 126 pb-1 $^{-1}$

$\mathrm{Q}^{2}>20 \mathrm{GeV}^{2}$

$c, b$ and If fractions extracted from a simultaneus fit of 3 discriminating variables:

$\mathrm{P}_{\mathrm{T}}^{\mathrm{rel}}, \delta, \mathrm{p}_{\mathrm{T}}^{\mathrm{miss} \| \mu}$

Theory : FFNS NLO (HVQDIS)

Charm : Good description

Beauty: Excess at low $Q^{2}(\sim 2 \sigma)$

\section{DESY-09-056}

\section{ZEUS}
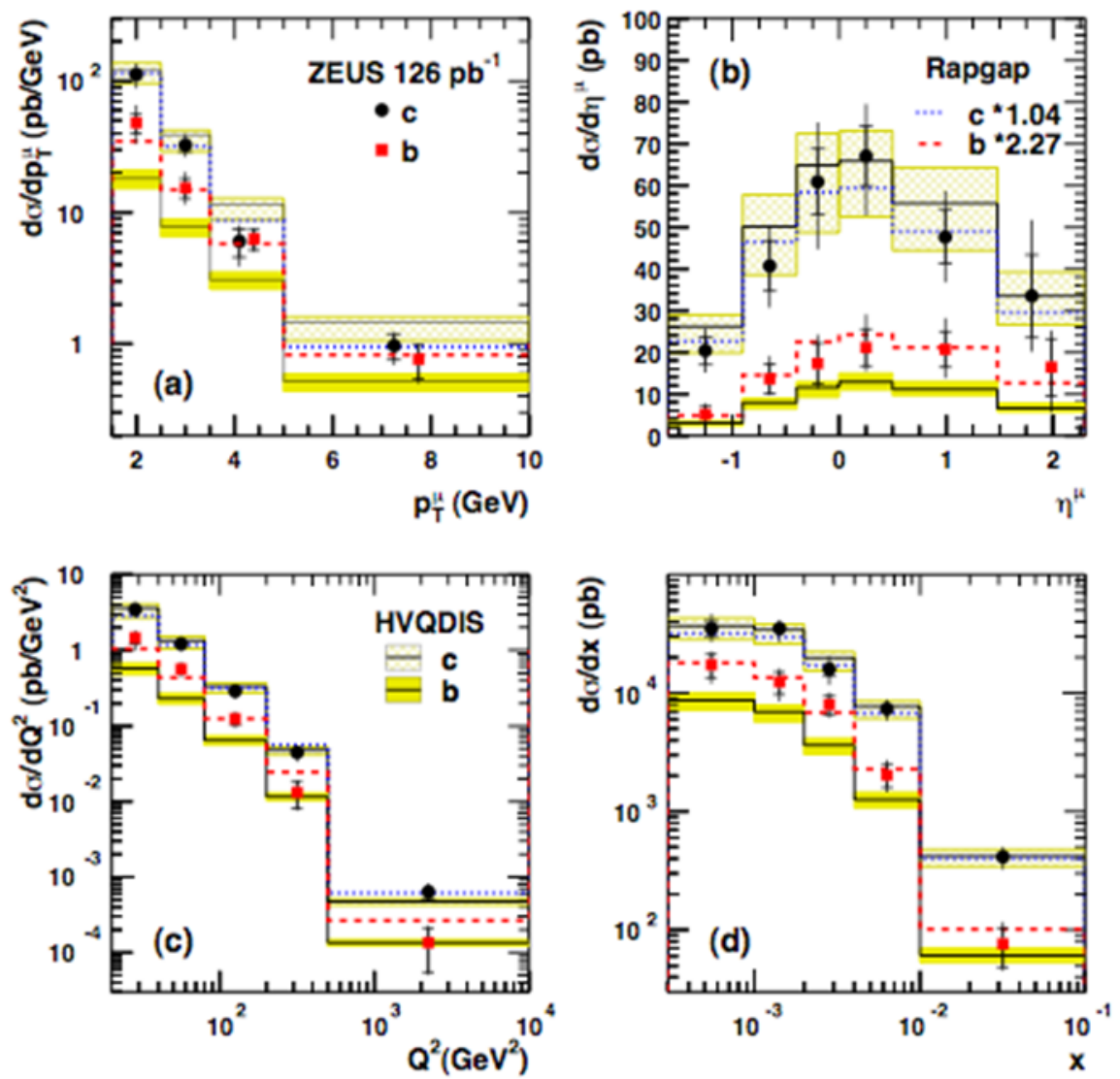

see P. Roloff[168], M. Bindi[179] 


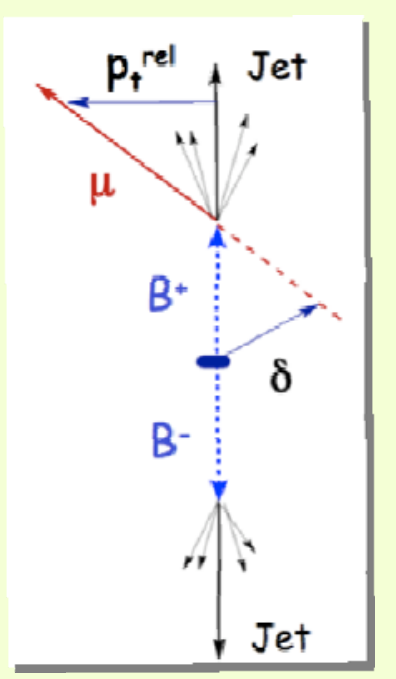

HERA II data $\left(05 \mathrm{e}^{+} \mathrm{p}\right) 125 \mathrm{pb}^{-1}$

Look for $b$ in two jets events with high-pt lepton

Kt clust:

$\mathrm{N}_{\text {jet }} \geq 2, \mathrm{P}_{\mathrm{t}}>7(6) \mathrm{GeV},|\eta|<2.5$

Theory: FMNR

Good agreement with NLO QCD

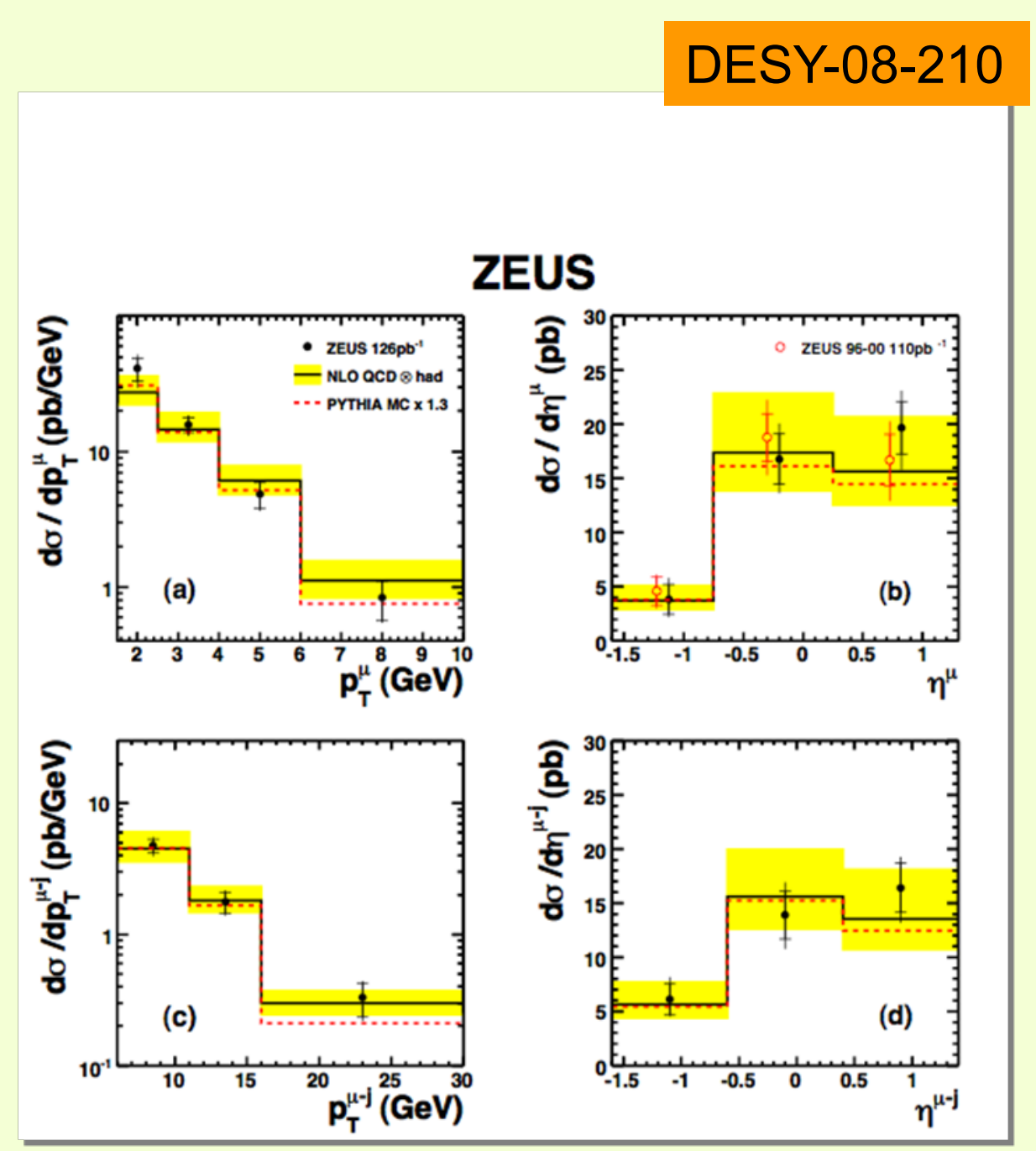




\section{Beauty in Photoproduction}

Lifetime tagging: Fraction of $b$ from decay length significance

Good agreement with NLO QCD
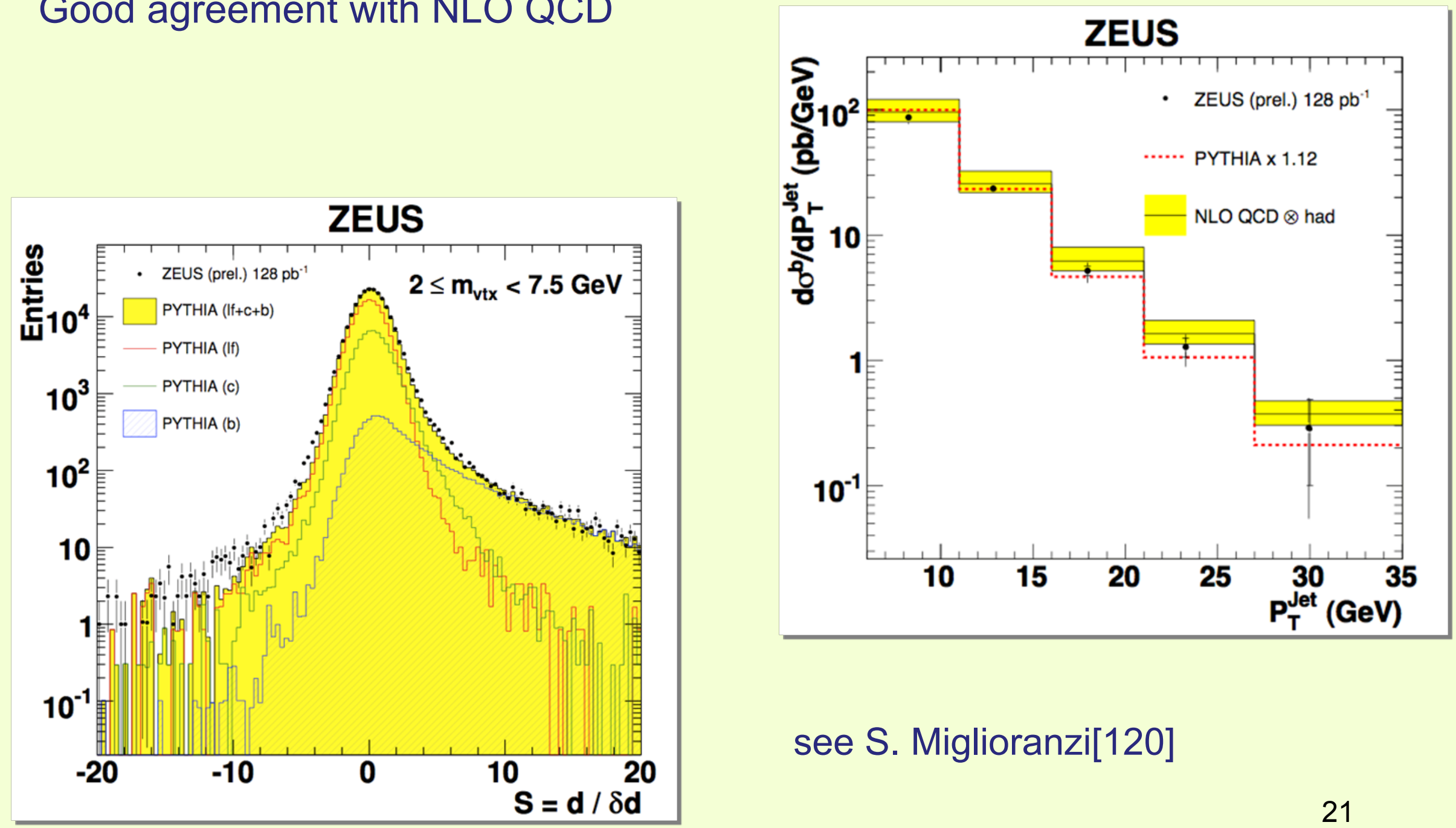

see S. Miglioranzi[120] 


\section{Beauty: Summary plot}

\section{HERA}

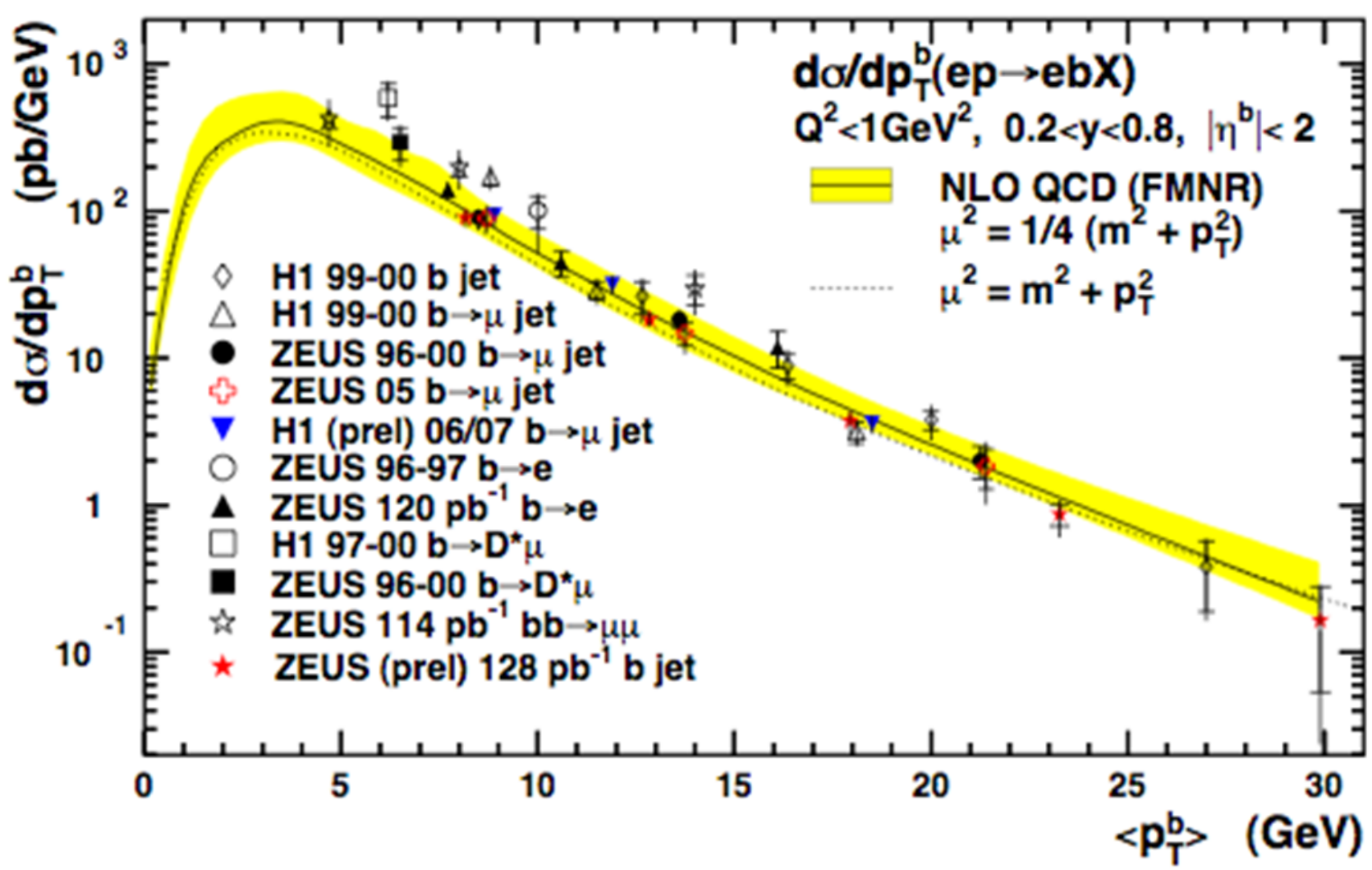




\section{Summary}

- Inclusive Measurements and Proton Structure

- Steady progress in the H1/ZEUS combination

- First $F_{L}$ measurement from ZEUS completed

* Huge effort to complete high-Q $\mathrm{Q}^{2}$ cross sections

$=>$ impact on the new ZEUS PDFs

- Exclusive processes and $\mathrm{pQCD}$

- New precise results on Jet and heavy quarks production

* We need better theory here

It has been a productive year for ZEUS

(18(6) papers 2008(9), 20 presentations to this Workshop)

But a lot of work (and fun!) analyising HERA II data is still

waiting us... 